\title{
Fluorescence Diagnostics of Colon Malignant and Premalignant Lesions Using 5-Aminolevulinic Acid
}

\author{
Elena V. Filonenko, ${ }^{1}$ Andrey D. Kaprin,, ${ }^{1}$ Antonina A. Raszhivina, ${ }^{2}$ \\ Antonina N. Urlova, ${ }^{1}$ and Andrey M. Nechipai ${ }^{2}$ \\ ${ }^{1}$ P. A. Herzen Moscow Cancer Research Institute, 2nd Botkinskiy Proezd 3, Moscow 125284, Russia \\ ${ }^{2}$ Department of Endoscopy, Russian Medical Academy for Postgraduate Training, 2nd Botkinskiy Proezd 5, Moscow 125284, Russia
}

Correspondence should be addressed to Elena V. Filonenko; derkul23@yandex.ru

Received 8 November 2013; Accepted 26 December 2013; Published 5 February 2014

Academic Editor: Victor Loschenov

Copyright (C) 2014 Elena V. Filonenko et al. This is an open access article distributed under the Creative Commons Attribution License, which permits unrestricted use, distribution, and reproduction in any medium, provided the original work is properly cited.

\begin{abstract}
Improvement of colon cancer diagnosis is a very important medical problem. Methods of fluorescence diagnosis (FD) with 5aminolevulinic acid (5-ALA) have been used in detection of early cancer on the surface of mucosa. The objective of the study was to estimate the effectiveness of FD with 5-ALA in the detection of malignant lesions in the colon. From 2008 to 2010 full examinations have been performed in 78 patients suffering from colon polyps. For the study we used drug Alasens based on 5ALA. Fluorescence colonoscopy with Alasens was made using the visual assessment of fluorescence images in combination with local fluorescence spectroscopy. The results of FD were compared with those of final histopathological study. The application of the method during colonoscopy allows specifying diagnosis, identifying areas of colon tumors transformation, and also showing a place for targeted biopsy. Sensitivity and specificity of FD with Alasens were $94.9 \%$ and $62.5 \%$, respectively. The application of local fluorescence spectroscopy as a step of the combined approach allows increasing the specificity of fluorescence colonoscopy from $62.5 \%$ up to $93.7 \%$.
\end{abstract}

\section{Introduction}

The accurate and early diagnosis of colon cancer provides the success of surgical and combined modality treatment, allows increasing the rate of good outcomes, extends the life expectancy, and improves the quality of life in cancer patients. Therefore, improvement of colon cancer diagnosis is a very important medical problem.

The colorectal cancer is known to arise from adenomatous polyp; thus early endoscopic diagnosis and treatment of such tumors are an effective method of colon cancer prevention [1]. According to literature data, adenomatous polyps are the origin of malignant tumors in $4-10 \%$ of cases, and true colon neoplasms are of a great concern. The main difficulties for diagnosis of early cancer developed from colon adenomas include the lack of specific presentation and pathognomonic features obtained by the instrumental examination and possible missing of colon adenomas, particularly flat and depressed adenomas [2]. Diagnostic possibilities of routine colonoscopy to identify such pathology are limited as there are no reliable visual criteria for neoplastic transformation of adenomas, and verification of endoscopic diagnosis based only on biopsy using forceps is successful only in $30-50 \%$ of cases [3].

Advanced endoscopic instruments may therefore decrease the miss rate of adenomas and optimize the potential for colorectal cancer prevention. One of these novel technologies is autofluorescence imaging (AFI), which can capture fluorescence $(500-630 \mathrm{~nm})$ emitted from intestinal or other tissues. The Japanese group of authors [4] showed that AFI increased the detection rate and reduced the miss rate of colorectal adenoma, particularly flat and depressed adenomas. However, this advantage of AFI was limited to the less-experienced endoscopists. The review of the available literature on the use of AFI for the diagnosis of colorectal neoplasms [5] also showed that the utility of AFI for "optical 
diagnosis" is limited. This may be due to the low resolution of current AFI video-endoscopy systems or to the subjective nature of interpreting color density of the images.

Therefore development of new methods for early diagnosis of colon polyp cancer transformation is necessary. One of such methods is fluorescence diagnosis (FD) with 5aminolevulinic acid (5-ALA).

Tumor cells are known to accumulate increased levels of photoactive protoporphyrin IX (PpIX) in presence of exogenous 5-ALA. This can be explained by a greater enzymes activity in the initial stages of heme synthesis and by lack of ferrochelatase, an enzyme turning PpIX into photoinactive heme [6]. This form of heme cannot be detected by fluorescence. Ferrochelatase activity is decreased in tumor cells due to limited iron availability and is considered to be the main cause of selective fluorescence of PpIX in tumor by many authors $[7,8]$.

5-ALA is used both for local and systemic application. Increased fluorescence of 5-ALA-induced PpIX in tumor compared with normal tissue allows identifying the areas of high PpIX accumulation, detecting occult precancer and early cancer foci, and outlining localization of different tumors. Sensitivity and specificity of the method according to different authors are ranging from $43 \%$ to $100 \%$ and from $51 \%$ to $96 \%$, respectively, depending on the tumor localization [9-16].

In the study of Bulgarian group of authors [17] exogenous fluorescence spectroscopy of esophageal and stomach tumors in vivo after oral application of 5-ALA six hours before spectroscopic measurements and video visualization of the patients using excitation at $405 \mathrm{~nm}$ was described. For rectal cancer there are also studies with other agents. For example, in the pilot study of German authors hexaminolevulinatebased fluorescence endoscopy was shown to be useful for detection of premalignant lesions. The study included ten patients with known rectal adenoma or cancer.

Thus, we decided to estimate the effectiveness of fluorescence diagnosis with 5-ALA in the detection of malignant lesions in the colon.

\section{Materials and Methods}

From 2008 to 2010 full examinations have been performed in 78 patients suffering from colon polyps. Twenty-seven (35\%) patients were men and $51(65 \%)$ were women. The age of patients was from 52 to 80 years. The mean age was $64.0 \pm 8.3$ years. The plan of full examination included clinical and biochemical blood analysis, clinical urine analysis, blood tests for syphilis, hepatitis B virus antigen, human immunodeficiency virus, electrocardiography, abdominal ultrasound, colonoscopy with biopsy using forceps, and FD. At the end of the examination, an endoscopic polypectomy was performed. The excised specimens were submitted to histopathological study.

For FD we used the photosensitizer Alasens (Research Institute of Organic Semi-Finished Products and Dyes, Moscow, Russia) given per os in the form of aqueous solution at a dose of $20-30 \mathrm{mg} / \mathrm{kg}$ body weight $3 \mathrm{~h}$ before fluorescence colonoscopy.

FD included colon examination in fluorescent mode. After endoscopic excision, polyps were reexamined in fluorescent mode and local fluorescence spectroscopy of the polyp's surface was performed in all cases.

For imaging under blue light excitation we used equipment produced by companies "Karl Storz" (Tuttlingen, Germany), "Olympus" (Japan). Video-assisted fluorescent lightemitting diode device UFF-630/675-01 produced by company "BIOSPEK" (Moscow, Russia) was used during the endoscopy. Laser electron-spectrum analyzer for FD (LESA01) by "BIOSPEK" (Moscow, Russia) with a wavelength of laser source at $632.8 \mathrm{~nm}$ and recoding spectrum of fluorescence in the range from 635 up to $800 \mathrm{~nm}$ was used to register local fluorescence spectrum.

During local fluorescence spectroscopy 5-65 spectra were recorded in every patient. From 5 to 65 spectra were registered and recorded for each patient during the local fluorescence spectroscopy; 2615 spectra of fluorescence were registered and analyzed. The values of diagnostic parameter (DP) were calculated and recorded in all cases. The values of DP were taken from several parts of the neoplasm under visual detection of fluorescence; after that the average value was calculated for each tumor. The fluorescence intensity of polyp tissues was estimated by DP, which was calculated automatically by a special program as a ratio of the peak area of PpIX (690-720 $\mathrm{nm})$ to the area of the reflected laser light (620-640 nm) and represented indirectly evidence of the Alasens-induced PpIX accumulation in the tissues. Fluorescence spectra of Alasens-induced PpIX in the intact colon mucosa were recorded with the determination of the fluorescent contrast of neoplastic/normal mucosa.

Malignant and premalignant (dysplasia) lesions with no fluorescence were defined as false-negative and benign tumors with fluorescence were defined as false-positive. Malignant and premalignant lesions with fluorescence were defined as positive and benign lesions without fluorescence were defined as negative.

\section{Results and Discussion}

3.1. Morphologic and Histopathological Findings. According to results of endoscopy $86 \%$ of all polyps were located in the lower parts of the left colon (rectum, rectosigmoid part, and sigmoid colon) and $14 \%$ were located above this level (descending colon, transverse colon, ascending colon, and caecum). One tumor was removed in 71 patients, two tumors in 5 patients, and 3 tumors in 2 patients. Totally, 87 tumors were diagnosed in 78 patients. According to the final histopathological study hyperplastic polyps were diagnosed in $9(10.3 \%)$ cases, benign adenomas in $39(44.8 \%)$, adenomas with different grades of dysplasia in 23 (26.4\%), and malignant adenomas in $16(18.5 \%)$ of the cases (Table 1).

3.2. Fluorescence Diagnosis. In 55 (63.2\%) of 87 cases fluorescence of different intensity (from pale pink to bright red) was registered on the surface of the tumors and in 32 (36.8\%) cases 
TABLE 1: Distribution of tumors depending on the results of visual fluorescence detection (FD) and final histopathological findings.

\begin{tabular}{|c|c|c|c|}
\hline \multirow{2}{*}{ Histopathological findings } & \multicolumn{2}{|c|}{ Results of visual FD } & \multirow{2}{*}{ Total } \\
\hline & $\mathrm{F}-$ & $\mathrm{F}+$ & \\
\hline \multicolumn{4}{|l|}{ Benign tumors } \\
\hline $\mathrm{HP}$ & - & 9 & 9 \\
\hline TA & 18 & 2 & 20 \\
\hline TVA & 4 & 3 & 7 \\
\hline VA & 8 & 4 & 12 \\
\hline Total & $30(62.5 \%)$ & $18(37.5 \%)$ & $48(100.0 \%)$ \\
\hline \multicolumn{4}{|c|}{ Adenomas with dysplasia I-III } \\
\hline TA dII & - & 5 & 5 \\
\hline TA dIII & - & 6 & 6 \\
\hline TVA dI & - & 3 & 3 \\
\hline TVA dII & - & 6 & 6 \\
\hline TVA dIII & - & 3 & 3 \\
\hline Total & - & $23(100.0 \%)$ & $23(100.0 \%)$ \\
\hline \multicolumn{4}{|l|}{ Malignant adenomas } \\
\hline HG AC & 1 & 7 & 8 \\
\hline MG AC & 1 & 7 & 8 \\
\hline Total & $2(12.5 \%)$ & $14(87.5 \%)$ & $16(100.0 \%)$ \\
\hline Total & $32(36.8 \%)$ & $55(63.2 \%)$ & $87(100.0 \%)$ \\
\hline
\end{tabular}

Note: F-: fluorescence was not detected; F+: fluorescence was registered; HP: hyperplastic polyp; TA: tubular adenoma; TA dII: tubular adenoma with grade II dysplasia; TA dIII: tubular adenoma with grade III dysplasia; TVA: tubular-villous adenoma; TVA dI: tubular-villous adenoma with grade I dysplasia; TVA dII: tubular-villous adenoma with grade II dysplasia; TVA dIII: tubular-villous adenoma with grade III dysplasia; VA: villous adenoma; HG AC: high-grade differentiated adenocarcinoma; MG AC: medium-grade differentiated adenocarcinoma.

had no fluorescence. Fluorescence was registered in $18(37.5 \%)$ of benign tumors, in $23(100 \%)$ of adenomas with dysplasia, and in $14(87.5 \%)$ of malignant adenomas. In 18 benign lesions with false-positive fluorescence 9 had inflammation origin and 12 were characterized by high proliferative activity. We suggested that $100 \%$ fluorescence in adenomas with dysplasia compared with benign tumors could be explained by high proliferative activity due to precancer process leading to malignant transformation [18].

The sensitivity of visual detection of fluorescence was $94.9 \%$ and the specificity $62.5 \%$.

The results of visual fluorescence detection in comparison with the results of routine histopathological investigation are shown in Table 1.

The spectra were registered in all areas of visual fluorescence. The values of DP were compared with the results of the routine morphological investigation. Distribution of tumors depending on the diagnostic parameter and the results of the final morphological investigation are presented in Table 2.

The level of Alasens-induced PpIX fluorescence on the surface of intact colon mucosa accounted for 0.20 to 0.86 arbitrary units (a.u.) (average, $0.56 \pm 0.25$ a.u.).

The DP ranged from 0.02 to 1.27 a.u. in $93.7 \%$ of benign tumors (average, $0.72 \pm 0.43$ a.u.). The average value of tumor/normal mucosa fluorescence contrast was $1.32 \pm$ 0.79 a.u. in the group of benign tumors. In three cases $(6.3 \%)$ the DP was $16.20,17.80$, and 26.84 a.u.; these values were defined as false-positive. All false-positive values were in patients with hyperplastic polyps.
TABLE 2: Distribution of tumors depending on the diagnostic parameter (DP) and final histopathological findings.

\begin{tabular}{lcccccc}
\hline Histopathological & \multicolumn{5}{c}{ Value of DP (a.u.) } & Total \\
findings & $0-1$ & $1-2$ & $2-3$ & $3-4$ & $>4$ & \\
\hline HP & & 6 & & & 3 & 9 \\
TA & 7 & 13 & & & & 20 \\
TVA & 3 & 4 & & & & 7 \\
VA & 6 & 6 & & & & 12 \\
TA dII & & & 5 & & & 5 \\
TA dIII & & 1 & 5 & & & 6 \\
TVA dI & 1 & 2 & & & & 3 \\
TVA dII & & & 6 & & & 6 \\
TVA dIII & & 1 & 2 & & & 3 \\
HG AC & & 1 & - & 2 & 5 & 8 \\
MG AC & & & 1 & 2 & 5 & 8 \\
Total & $\mathbf{1 7}$ & $\mathbf{3 4}$ & $\mathbf{1 9}$ & $\mathbf{4}$ & $\mathbf{1 3}$ & $\mathbf{8 7}$ \\
\hline
\end{tabular}

Note: HP: hyperplastic polyp; TA: tubular adenoma; TA dII: tubular adenoma with grade II dysplasia; TA dIII: tubular adenoma with grade III dysplasia; TVA: tubular-villous adenoma; TVA dI: tubular-villous adenoma with grade I dysplasia; TVA dII: tubular-villous adenoma with II dysplasia; TVA dIII: tubular-villous adenoma with grade III dysplasia; VA: villous adenoma; HG AC: high-grade differentiated adenocarcinoma; MG AC: medium-grade differentiated adenocarcinoma.

In the group of tumors with different degrees of dysplasia, DP values ranged from 0.76 to 2.73 (average, $1.83 \pm 0.70$ a.u.). The average value of fluorescence contrast tumor/normal in this group was $3.33 \pm 1.26$ a.u. 
In a group of patients with malignant colon adenomas the DP ranged from 3.1 to 20.8 (average, $7.32 \pm 6.70$ a.u.) in $87.5 \%$ of cases. The average value of fluorescence contrast in this group was $13.32 \pm 12.17$ a.u. In two cases $(12.5 \%)$ the DP was 1.49 and 2.70 a.u.; these values were defined as false-negative.

For a threshold level set at 3 a.u., the sensitivity of the method of the local fluorescence spectroscopy was $84.6 \%$ and the specificity $93.7 \%$.

Thus, the study showed that sensitivity and specificity of fluorescence diagnosis with visual evaluation of fluorescence with Alasens were $94.9 \%$ and $62.5 \%$, respectively. For example, Messmann et al. showed sensitivity and specificity to be $43 \%$ and $73 \%$ [18]. Complementation of this method by local fluorescence spectroscopy allows increasing the specificity up to $93.7 \%$. The values of DP for normal mucosa were $0.56 \pm$ 0.25 , pre-cancer (dysplastic tissue) $-1.83 \pm 0.7$, and cancer$7.32 \pm 6.7$. These differences were significant (Mann-Whitney $U$-test) between normal mucosa and cancer $(P=.018)$ and normal mucosa and precancer (I-III grades of dysplasia) $(P=$ .043). Thus, combined method of fluorescence diagnosis is a highly informative method for detecting malignant transformation in colon adenomas. Visual fluorescence diagnosis allows detecting fluorescence area on surface of colon polyps that is indicative for increased proliferation or transformation in benign lesions; these areas are difficult to be found for routine colonoscopy without using fluorescence diagnosis. According to results of study colon lesions with fluorescence should be excised with further histopathological verification.

\section{Conclusions}

FD with 5-ALA is an effective method of colon cancer detection. The application of the method during colonoscopy allows specifying diagnosis, identifying areas of colon tumors transformation, and also showing a place for targeted biopsy. The local fluorescence spectroscopy as a highly informative diagnostic method increased specificity of fluorescence colonoscopy with 5-ALA from $62.5 \%$ to $93.7 \%$.

\section{Conflict of Interests}

The authors declare that there is no conflict of interests regarding the publication of this paper.

\section{References}

[1] P. Mlkvy, J. Majek, L. Jurgos, P. Makovnik, and S. Durdik, "Endoscopic treatment of praecancerous colorectal lesions and early colorectal cancer," Bratislavské Lekárske Listy, vol. 111, no. 1, pp. 50-53, 2010.

[2] S. Tsuda, B. Veress, E. Tóth, and F.-T. Fork, "Flat and depressed colorectal tumours in a southern Swedish population: a prospective chromoendoscopic and histopathological study," Gut, vol. 51, no. 4, pp. 550-555, 2002.

[3] V. V. Veselov, Endoscopic treatment of patients with large and giant colon adenomas [Doctoral dissertation], State Science Center of Coloproctology, Moscow, Russia, 1997.

[4] K. Moriichi, M. Fujiya, R. Sato et al., "Back-to-back comparison of auto-fluorescence imaging (AFI) versus high resolution white light colonoscopy for adenoma detection," BMC Gastroenterology, vol. 12, article 75, 2012.

[5] Y. Takeuchi, N. Uedo, M. Hanafusa et al., "Endoscopic diagnosis of colorectal neoplasms using autofluorescence imaging," Intestinal Research, vol. 10, no. 2, pp. 142-151, 2012.

[6] V. Manivasager, K. K. L. Yee, P. W. S. Heng, K. C. Soo, and M. Olivo, "A study comparing endogenous protoporphyrin IX induced by 5-ALA and ALA-methyl ester with exogenous PpIX and PpIX dimethyl ester in photodynamic diagnosis of human nasopharyngeal carcinoma xenografts," International Journal of Oncology, vol. 29, no. 4, pp. 997-1002, 2006.

[7] M. Triesscheijn, P. Baas, J. H. M. Schellens, and F. A. Stewart, "Photodynamic therapy in oncology," The Oncologist, vol. 11, no. 9, pp. 1034-1044, 2006.

[8] D. L. Campbell, E. F. Gudgin-Dickson, P. G. Forkert, R. H. Pottler, and J. C. Kennedy, "Detection of early stages of carcinogenesis in adenomas of murine lung by 5 -aminolevulinic acidinduced protoporphyrin IX fluorescence," Photochemistry and Photobiology, vol. 64, no. 4, pp. 676-682, 1996.

[9] W. J. Piotrowski, J. Marczak, A. Nawrocka, A. Antczak, and P. Górski, "Inhalations of 5-ALA in photodynamic diagnosis of bronchial cancer," Monaldi Archives for Chest Disease, vol. 61, no. 2, pp. 86-93, 2004.

[10] H. Stepp and R. Waldelich, "Fluorescence diagnosis and photodynamic therapy in urology," Aktuelle Urologie, vol. 38, no. 6, pp. 455-464, 2007.

[11] R. Baumgartner, R. M. Huber, H. Schulz et al., "Inhalation of 5-aminolevulinic acid: a new technique for fluorescence detection of early stage lung cancer," Journal of Photochemistry and Photobiology B, vol. 36, no. 2, pp. 169-174, 1996.

[12] H. Hautmann, J. P. Pichler, H. Stepp, R. Baumgartner, F. Gamarra, and R. M. Huber, "In-vivo kinetics of inhaled 5aminolevulinic acid-induced protoporphyrin IX fluorescence in bronchial tissue," Respiratory Research, vol. 8, no. 1, article 33, pp. 33-39, 2007.

[13] S. G. Piccirillo, S. Dietz, B. Madhu et al., "Fluorescence-guided surgical sampling of glioblastoma identifies phenotypically distinct tumour-initiating cell populations in the tumour mass and margin," British Journal of Cancer, vol. 107, no. 3, pp. 462468, 2012.

[14] E. V. Filonenko, V. V. Sokolov, V. I. Chissov, E. A. Lukyanets, and G. N. Vorozhtsov, "Photodynamic therapy of early esophageal cancer," Photodiagnosis and Photodynamic Therapy, vol. 5, no. 3, pp. 187-190, 2008.

[15] E. V. Filonenko, Fluorescence diagnosis and photodynamic therapy in oncology [Doctoral dissertation], P. A. Herzen Moscow Cancer Institute, Moscow, Russia, 2006.

[16] H. Stepp, R. Sroka, and R. Baumgartner, "Fluorescence endoscopy of gastrointestinal diseases: basic principles, techniques, and clinical experience," Endoscopy, vol. 30, no. 4, pp. 379-386, 1998.

[17] E. Borisova, B. Vladimirov, and L. Avramov, "5-ALA mediated fluorescence detection of gastrointestinal tumors," Advances in Optical Technologies, vol. 2008, Article ID 862081, 7 pages, 2008.

[18] H. Messmann, E. Endlicher, G. Freunek, P. Rümmele, J. Schölmerich, and R. Knüchel, "Fluorescence endoscopy for the detection of low and high grade dysplasia in ulcerative colitis using systemic or local 5-aminolaevulinic acid sensitisation," Gut, vol. 52, no. 7, pp. 1003-1007, 2003. 

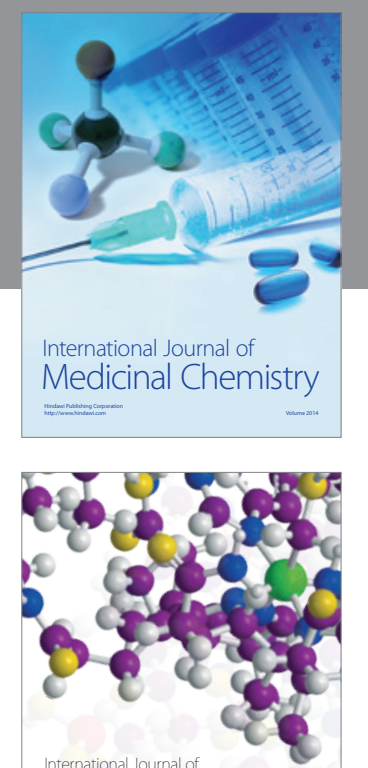

\section{Carbohydrate} Chemistry

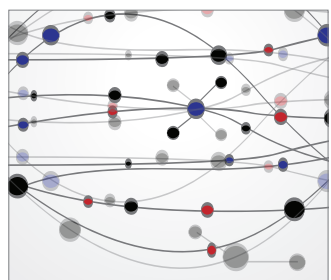

The Scientific World Journal
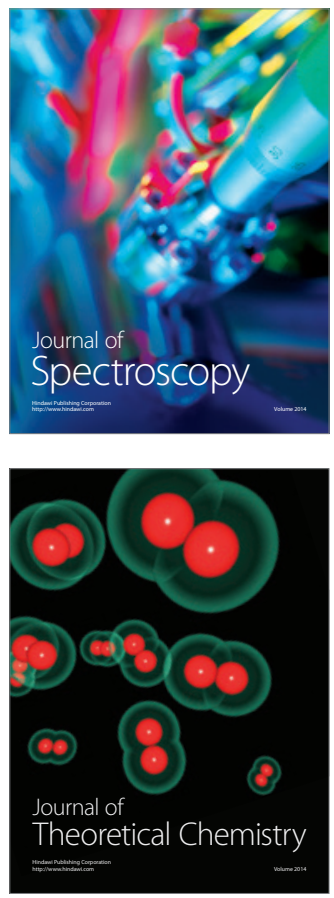
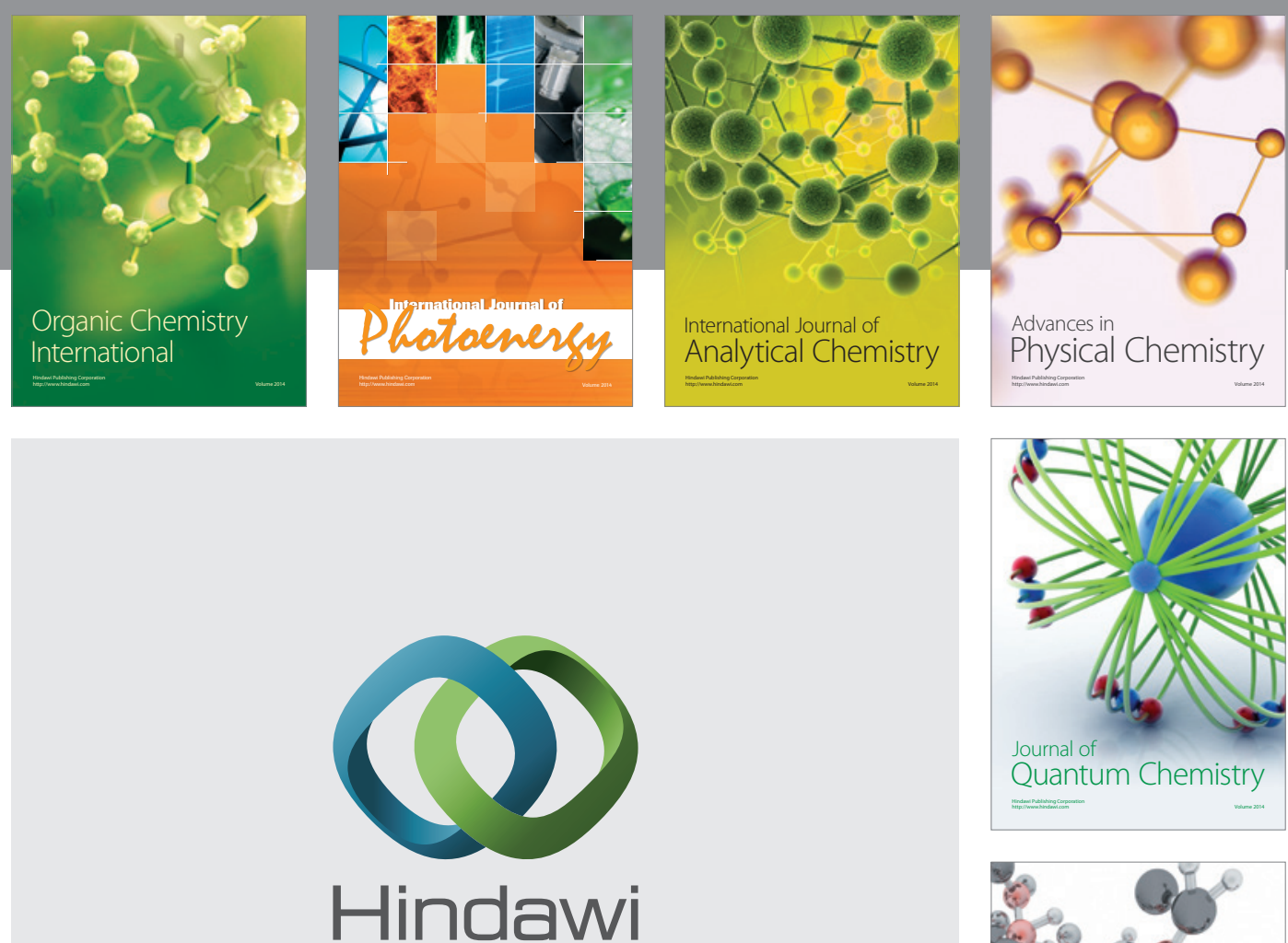

Submit your manuscripts at

http://www.hindawi.com

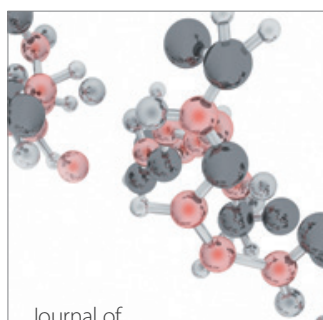

Analytical Methods

in Chemistry

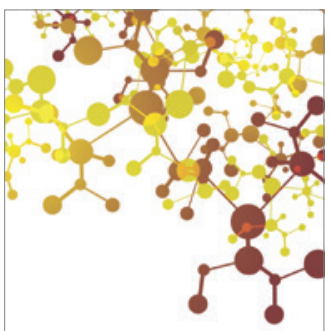

Journal of

Applied Chemistry

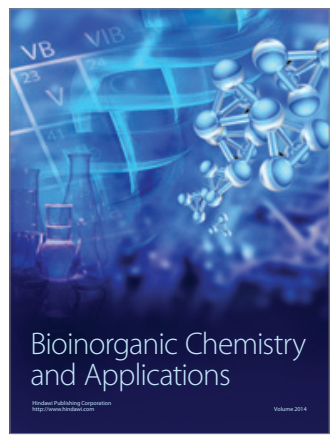

Inorganic Chemistry
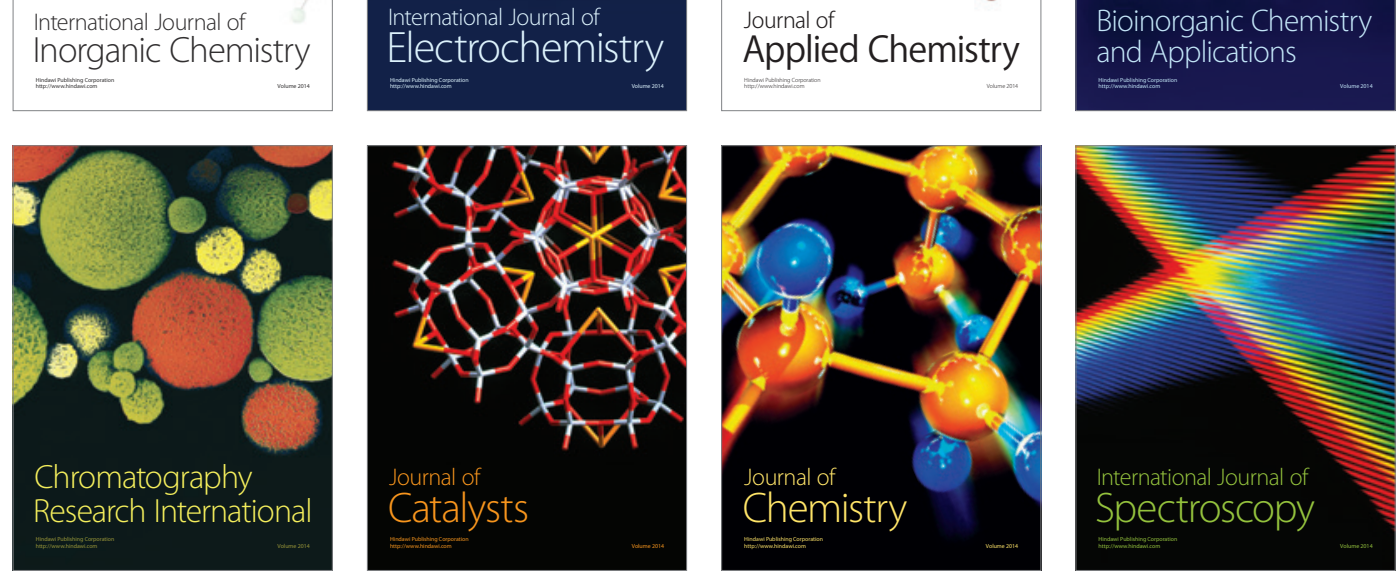